\title{
When central populations exhibit more genetic diversity than peripheral populations: A simulation study
}

\author{
DAI Qiang ${ }^{1,2} \&$ FU JinZhong ${ }^{3 *}$ \\ ${ }^{1}$ Chengdu Institute of Biology, Chinese Academy of Sciences, Chengdu 610041, China; \\ ${ }^{2}$ Department of Zoology, University of British Columbia, Vancouver, British Columbia V6T 1Z4, Canada; \\ ${ }^{3}$ Department of Integrative Biology, University of Guelph, Guelph, Ontario N1G 2W1, Canada
}

Received April 27, 2011; accepted May 27, 2011

\begin{abstract}
The central-peripheral population hypothesis $(\mathrm{CPH})$ predicts that peripheral populations have reduced genetic variability. Therefore, it is often assumed that they deserve higher conservation priority over central populations. We examined this hypothesis using computer simulations with the objective of determining the range of species properties (parameters) under which a species is likely to exhibit the $\mathrm{CPH}$ pattern. The interaction between migration, genetic drift, and time of population establishment was examined; in particular, various parameters of migration, such as mode of dispersal, migration rate, and maximum migration distance, were investigated. The $\mathrm{CPH}$ pattern was observed only within a narrow parameter window of various species properties. Active dispersers with low migration rate and moderate maximum migration distance were more likely to have higher genetic diversity in the central populations than in the peripheral populations. Newly established populations were also more likely to exhibit the $\mathrm{CPH}$ pattern. Although migration rate appeared to be the most important determining factor, sensitivity analysis suggested that the interaction between parameters is probably more important than any single parameter. Our findings have important implications for conservation programs.
\end{abstract}

central-peripheral population hypothesis, migration, genetic drift, population establishment time, active disperser, passive disperser, conservation

Citation: Dai Q, Fu J Z. When central populations exhibit more genetic diversity than peripheral populations: A simulation study. Chinese Sci Bull, 2011, 56: 2531-2540, doi: 10.1007/s11434-011-4605-x

The importance of peripheral populations for the evolution and conservation of a species has been a highly debated topic and many arguments are rooted in Mayr's centralperipheral population hypothesis $(\mathrm{CPH})$ [1]. This hypothesis states that a reduction in gene flow and increased selection pressure tends to deplete the genetic variability of peripheral populations. Peripheral populations are usually small and are often established by a few founders. Consequently, they often suffer more genetic drift. Peripheral populations are also isolated from the central populations, and therefore have reduced gene flow to counter genetic drift. Furthermore, habitats are often marginal near the species border and selection is severe, thus only a small number of geno-

*Corresponding author (email: jfu@uoguelph.ca) types survive. Peripheral populations are also likely to experience different selection regimes, and are expected to be genetically distinct from the central populations [2]. Although the theoretical predictions are straightforward, what actually happens in natural populations is much more complex. For example, selection at the periphery is often unpredictable; heterozygous advantage may be more pronounced in peripheral populations, which may lead to more genetic diversity [3]. Ecological marginal habitat may not be the same as geographic peripheral [4].

The hypothesis has important implications in conservation [2,5]. Many authors have argued that more divergent taxa or populations have greater conservation value, and peripheral populations are often assumed to be genetically and morphologically divergent from central populations [6]. 
Furthermore, the periphery of a species is often considered the most active region of diversification or speciation $[1,7,8]$. Currently, large resources are spent on protecting geographically peripheral populations of widespread species, although many species are common elsewhere within their distribution $[2,9,10]$.

Numerous empirical studies have been carried out to test the hypothesis. Many of the studies support the $\mathrm{CPH}$ pattern while others rejected it [2]. Lönn and Prentice [11] found a correlation between genetic variability and population location relative to its range periphery in a perennial herb (Gypsophila fastigiata) and explained the results using a combination of genetic drift and lower levels of inter-population gene flow. Durka [8] reported similar results in an annual herb species (Corrigiola litoralis). In animals, Cassel and Tammaru [10] found low diversity in both peripheral and isolated populations of a butterfly species (Coenonympha hero) and Zhan et al. [12] reported low genetic diversity in 2 peripheral populations of the Chinese wood frog (Rana chensiensis). Several explanations have been proposed for studies that rejected the hypothesis. In pitch pine, it was suggested that phenotypic plasticity might compensate for the variation in microhabitats without alleles being lost [13]. Several studies found high levels of gene flow and a selection regime that favored heterozygotes which promoted genetic divergence in peripheral populations of, such as Pinus edulis [14] and Sitka spruce [5]. Garner et al. [15] found an east-to-west gradient of declining diversity in the Italian agile frog (Rana latastei) irrespective of how peripheral the populations were. It was suggested that the range of the frog was established after rapid post-glaciation expansion from a Balkan refugium and that the genetic diversity might be determined more by the location relative to the expansion source than to core of the geographic range.

The contradictory results from empirical studies have highly undervalued the predictive power of the hypothesis. The disagreements may result from the undefined nature of the parameters of the hypothesis. Different species have vastly different dispersal abilities, histories and resilience to selection; therefore, they may or may not follow the predictions of the central-peripheral difference in genetic diversity. The accumulation of even more case studies is unlikely to resolve the dispute. Rather, the parameters, within a certain range of which the peripheral populations are likely to exhibit lower genetic diversity than the central populations, should be determined and tested. Lesica and Allendorf [2] suggested 4 factors that should be considered: (i) spatial distance (degree of isolation); (ii) life history (poor dispersal ability); (iii) time of population establishment; and (iv) ecology (directional selection). Using a modeling approach, Garcia-Ramos and Kirkpatrick [16] examined the interactionbetween directional selection and gene flow, and concluded that the disruption of gene flow could cause rapid divergence of the peripheral populations even in the absence of genetic drift. Such theoretical approaches will help fine-tune the $\mathrm{CPH}$ and may be more fruitful than case studies.

In this study, we examined the interaction between migration (dispersal), genetic drift, and time of population establishment using computer simulations. We particularly focused on various parameters of migration, such as mode of dispersal, migration rate and maximum migration distance. Our objective was to determine the range of various species characteristics (parameters) under which the peripheral populations are likely to exhibit lower genetic diversity than the central populations. With well-defined parameters, the central-peripheral population hypothesis will have more predictive power and will be able to provide more accurate guidelines for the conservation community.

\section{Materials and methods}

\subsection{Experimental design}

We mainly tested the interaction between 3 evolutionary forces in shaping the genetic diversity of the central and peripheral populations: genetic drift, migration and the time since population establishment. The effective population size $\left(N_{\mathrm{e}}\right)$ was used to represent the strength of genetic drift, and the number of generations was used to measure the time since population establishment. A significant part of the European and North American fauna and flora reached their current distribution ranges after the last Pleistocene glaciation, approximately 10000 years ago [17,18]. Therefore, simulations were run for a total of 10000 generations with the intention of simulating the post-glaciation timeframe. Assuming a generation time of one year, 10000 generations would approximate the full duration of post-glaciation time. Three parameters for migration were considered: mode, rate and maximum distance. Two modes of migration were categorized, active dispersal and passive dispersal. A passive disperser is defined as an organism whose movement is completely controlled by media, for example, a seed carried by wind. Active dispersers, for example, most vertebrates, control their movement, at least partially. In our simulation model, a passive disperser was one that died if it went out of its distribution range (suitable habitat), and an active disperser was one that retreated back to its range to continue its migration. Migration rate $(m)$ was defined as the proportion of a population leaving the parental population per generation. The maximum migration distance $\left(D_{\mathrm{m}}\right)$ was the maximum movement distance during an individual's life time. In the simulation runs, we varied this distance from $1 \%$ to $150 \%$ of the radius of the distribution. Some large marine turtles, for example, may move much longer distance than their distribution in one generation. The actual individual dispersal distance followed a triangular distribution. For a triangular distribution with minimum $L$, mode $M$ and maximum $U$, the probability density function (PDF) is given by 


$$
P(x \mid L, M, U)= \begin{cases}\frac{2(x-L)}{(U-L)(M-L)}, & L \leqslant x \leqslant M \\ \frac{2(U-x)}{(U-L)(U-M)}, & M \leqslant x \leqslant U, \\ 0, & \text { otherwise }\end{cases}
$$

where $x$ is the actual dispersal distance, and $L, M, U$ are the lower limit, upper limit and mode of actual dispersal distance, respectively. In our simulation, $L$ and $M$ were set to 0 , meaning that short dispersal distances are more frequent than longer ones with average dispersal distances of $U / 3$.

The contribution of mutations was also considered and the mutation rate was set to $10^{-7}$. Only forward mutations were included and it was assumed that mutations would produce new alleles. Selection parameters were not included because a substantial amount of attention has already been paid to the interaction between genetic drift and natural selection [2]. Previous studies have suggested that selection in the peripheral population is often unpredictable rather than clinal because selection was shown to either increase or decrease the genetic diversity [2,19]. Furthermore, empirical testing of the results of this simulation with neutral genetic markers such as microsatellite DNA loci, which are presumed to be neutral, will not require parameterization of selection pressure. Finally, expected heterozygosity $\left(H_{\mathrm{e}}\right)$ was used to measure genetic diversity.

\subsection{Simulation parameters}

The simulation was conducted in the hypothetical space of a circle with a radius of 100 , similar to the island model. All populations were diploid, sexually reproducing and had no overlapping generations. A total of 100 populations were randomly distributed in this space, and the location of each population remained the same during each simulation run.

Genetic data from 100 loci were examined and all loci were assumed to be in linkage equilibrium. For each locus, the number of alleles was set to 20 with an equal initial frequency $\left(P_{1}=P_{2}=\cdots=P_{20}=0.05\right)$ and each mutation created a new allele. All individuals were heterozygous $\left(H_{\mathrm{e}}=\right.$ 0.95 ) in the initial generation.

Population size was varied in two ways. First, all populations had the same size (population size constant). Three different population sizes, 50, 100, 500, were examined in the simulations. Second, peripheral populations had smaller sizes (population size decline). The population size was assumed to decline linearly with distance from center of the space. The size of a population is given by $N_{c}(1-d / 200)$, where $N_{\mathrm{c}}$ is the size of the center population and $d$ is the distance from the center. Three different sizes of the center population, 50, 100, 500, were examined in the simulations. The effective population size remained the same from generation to generation.

Four migration rates $(m ; 0.00001,0.0001,0.001$, and
$0.01)$ and 5 maximum migration distances $\left(D_{\mathrm{m}} ; 1,10,50\right.$, 100 , and 150) were examined. Migration occurred after reproduction. It was assumed that more individuals were being reproduced in each generation than the carrying capacity (effective population size) of the population. Some individuals died, some emigrated and the rest remained in the population to keep effective population size the same as in previous generations. Migration rate of every population was $m$ and the actual number of migrants from every population followed a Poisson distribution. The genotype of an emigrant was selected by a random draw from the parental population. Emigration did not change the effective population size or the allele frequencies of the parental population in the next generation. Emigrants dispersed in random directions following a uniform distribution, while their dispersal distance followed a triangular distribution. At the end of dispersal, a migrant would join the nearest population with the exception of its parental population. If a migrant dispersed out of the circular space (species distribution range), an active disperser would turn back and continue the journey before eventually joining the nearest population and a passive disperser would disappear from our simulation. Each emigrant will replace an individual from its destination population; thus, the allele frequency of a population would change after the population accepted a migrant but its effective size would remain the same.

A summary of the simulation parameters used in this study is presented in Table 1 . The values for both population size and migration rate are relatively small [20]. With the current global loss of biodiversity, small populations in a fragmented landscape are of particular concern within the conservation community. In addition, small populations are more likely to be sensitive to migration, and hence allow a more accurate evaluation of the impact of migration on the distribution of genetic diversity. Furthermore, a high migration rate would almost certainly homogenize the populations, and therefore, only low migration provides opportunities to test the predictions of the $\mathrm{CPH}$.

Ten repeats for each parameter combination were conducted. At the end of each simulation run, the expected heterozygosity was calculated for each population. A linear regression was performed between the expected heterozygosity $(Y)$ of each population and the distance from the space center $(X)$ to obtain the slope. A negative value for the slope indicates that the expected heterozygosity of populations decreased with distance from the space center and the absolute value of the slope is a measure of how quickly the expected heterozygosity of populations changed with distance.

\subsection{Sensitivity analysis}

Sensitivity analysis has recently gained popularity as the method of choice to evaluate the importance of each parameter to the prediction and to assess how the parameters 
Table 1 Summary of the simulation parameters used in this study

\begin{tabular}{cc}
\hline & Parameter range \\
\hline $\begin{array}{c}\text { Simulation space (species distribu- } \\
\text { tion) }\end{array}$ & A circular area with a radius of 100 \\
Number of populations & 100 \\
Central population size $\left(N_{\mathrm{c}}\right)$ & 50,100 and 500 \\
Population size variation & (2) Sizes decline with distance from \\
& center \\
Migration rate $(m)$ & $0.00001,0.0001,0.001$, and 0.01 \\
Maximum migration distance $\left(D_{\mathrm{m}}\right)$ & $1,10,50,100$, and 150 \\
Mode of dispersal & $(1)$ Active dispersers \\
Number of loci & $(2)$ Passive dispersers \\
Mutation rate & 100 \\
Initial number of alleles per locus & 20 \\
Mutation & $10^{-7}$ per generation per locus \\
\hline
\end{tabular}

affect the behavior of the model [21,22]. We used a sequential global sensitivity analysis to determine the importance of all the parameters over time. For every 1000 generations, the sensitivity index of a parameter was estimated from an ANOVA sum of squares for the parameter based on the outputs of the mean $H_{\mathrm{e}}$ and the mean slope of the linear regression [23,24]. The two sets of indices for $H_{\mathrm{e}}$ and for the slope were calculated separately. Furthermore, we used a generalized sensitivity index to measure the overall contribution of each parameter [24]. All analyses were performed with package "multisensi" [25] in $R$ [26].

\section{Results and discussion}

\subsection{The impact of genetic drift and migration on $H_{e}$}

The average expected heterozygosity $\left(H_{\mathrm{e}}\right)$ of the 100 populations for each parameter combination after 10000 generations are shown in Figure 1. In Figure 2, the $H_{\mathrm{e}}$ of each population for each parameter combination after 10000 generations is presented. Not surprisingly, many populations finished with $H_{\mathrm{e}}$ equal to zero. This was particularly so when population sizes were small $\left(N_{\mathrm{e}}=50-100\right)$ and migration rates were extremely low ( $m=0.00001-0.0001$; Figures 1 and 2). This result is consistent with the classic view that small populations cannot sustain their genetic diversity [27]. Furthermore, simulation runs with larger population sizes consistently finished with higher $H_{\mathrm{e}}$ (Figure 1). This is likely driven by genetic drift. Whether the peripheral populations had equal or smaller sizes than the central populations had little impact on the final $H_{\mathrm{e}}$ values. The patterns

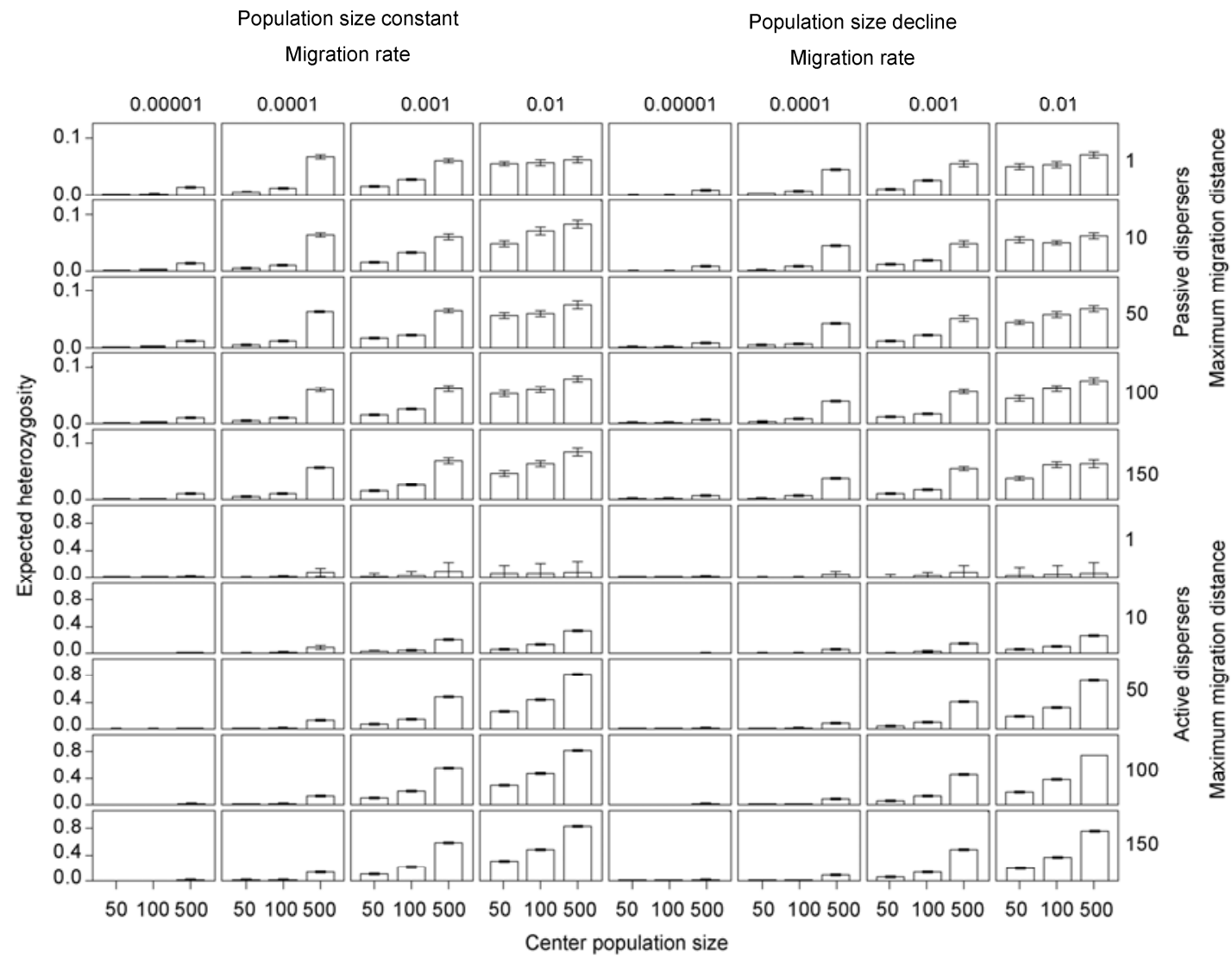

Figure 1 Average expected heterozygosity $\left(H_{\mathrm{e}}\right)$ of 100 populations for each parameter combination after 10000 generations. Each simulation was repeated 10 times and data were pooled. Error bar shows \pm 1.0 standard error (SE). Note that the scales on the $y$-axes are different in the top 5 rows compared to the bottom 5 rows. 


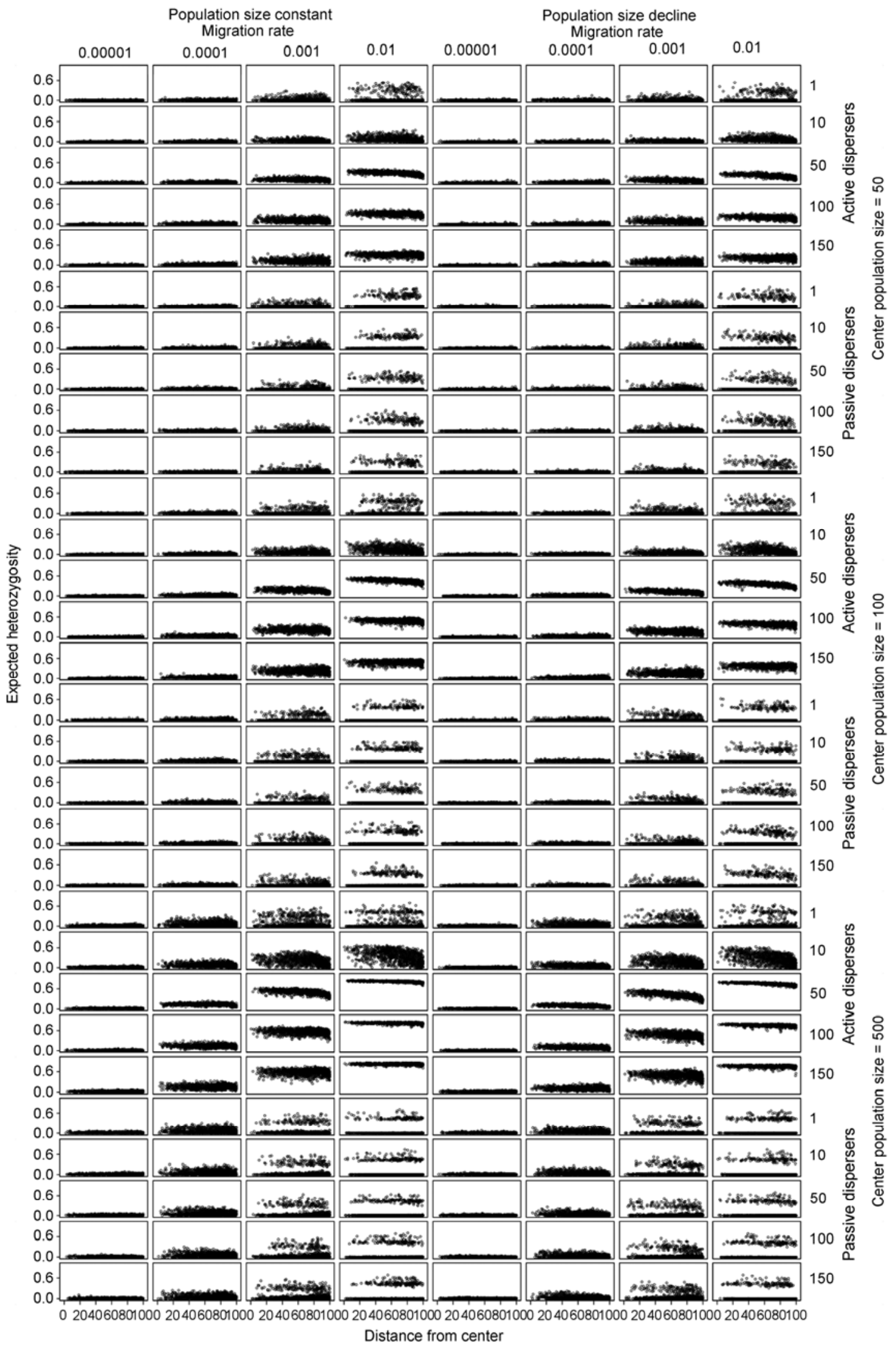

Figure 2 Expected heterozygosity $\left(H_{\mathrm{e}}\right)$ of each population after 10000 generations. Each simulation was repeated 10 times and the data were pooled. Each circle represents one population and there were 100 populations in each simulation. Times and data was regressed separately. Error bar shows \pm 1.0 standard error (SE). 
for the two sets of simulations were nearly identical, although the simulations produced slightly higher $H_{\mathrm{e}}$ when the peripheral populations had equal size to the central populations (Figures 1 and 2). This result is also likely to be caused by stronger genetic drift in the smaller peripheral populations.

It was surprising that even a small migration helped populations maintain their genetic diversity. With $m=0.001-$ 0.01 and $D_{\mathrm{m}} \geqslant 50$, all populations of active dispersers and a large number of populations of passive dispersers maintained their diversity (Figure 2). More migration, via higher migration rates, larger maximum distances or both, consistently produced higher $H_{\mathrm{e}}$ (Figures 1 and 2). This result is congruent with the classic population genetic theory. The mode of migration also appeared to have a significant impact on the $H_{\mathrm{e}}$ values. Everything else being equal, active dispersers consistently had higher $H_{\mathrm{e}}$ values than the passive dispersers (Figure 1). While a large portion of passive dispersers' populations reached homozygosity $\left(H_{\mathrm{e}}=0\right)$ after 10000 generations, some populations of active dispersers reached homozygosity only when their migration ability was very low, e.g. $m \leqslant 0.0001$ and $D_{\mathrm{m}}=1$ (Figures 2 and $\mathrm{S} 1$ ). Additionally, active and passive dispersers responded differently to changes in the maximum migration distance. For active dispersers, $H_{\mathrm{e}}$ increased with the increase of $D_{\mathrm{m}}$ until $D_{\mathrm{m}}$ reached 50, i.e. until the average migration distance of the migrants reached 50/3. After that, $H_{\mathrm{e}}$ remained more or less the same (Figure 1). This is also consistent with the classic population genetic theory. When $D_{\mathrm{m}}$ is small, gene flow only occurs between populations in close proximity and, therefore, is limited; when $D_{\mathrm{m}}$ becomes larger, gene flow increases, producing high $H_{\mathrm{e}}$. However, when $D_{\mathrm{m}} \geqslant 50$, gene flow is no longer limited by migration distance and further increases of $D_{\mathrm{m}}$ will not improve gene flow. For passive dispersers, the change of $D_{\mathrm{m}}$ had little impact on $H_{\mathrm{e}}$ (Figure 1). This is likely because a larger $D_{\mathrm{m}}$ increases not only the movement between populations but also the number of individuals that may disperse out of the simulation area (and die). Consequently, the increase of $D_{\mathrm{m}}$ of passive dispersers does not significantly increase "effective migration" and gene flow.

Sensitivity analysis confirmed our observation (Figures 3(a) and 4(a)). The two genetic drift related parameters, effective population size and whether or not population size declined from the center, were significant at early stages of the simulation but their importance decreased over time. On the other hand, the importance of the three migration-related parameters was small at the beginning but increased over time. The largest changes occurred in the first 4000 generations. Clearly, the effect of migration only became important after genetic drift created sufficient population heterogeneity. Overall, migration rate and migration mode were the most important parameters, largely because of their interaction with each other and with other parameters. Whether or not population size decline from the center only had a minor impact on the final outcome of $H_{\mathrm{e}}$.

\subsection{The impact of genetic drift and migration on the regression slope}

Figure 5 shows the mean slopes of the linear regression analysis on $H_{\mathrm{e}}$ and distance of 100 populations for each parameter combination after 10000 generations. A negative value indicates that the central populations had higher genetic diversity $\left(H_{\mathrm{e}}\right)$ than the peripheral populations. Clearly, the majority of scenarios did not show a negative value. Of the 240 sets of parameter combinations studied, only 73 were negative and clearly different from zero (Figure 5).

Migration rate and migration mode appeared to have the most significant impact on the outcome of the regression slope, and they showed interesting interactions with maximum migration distance. A migration rate as low as 0.0001 produced a significant and consistent negative slope when $N_{\mathrm{e}}=500$ and population size decline from the center
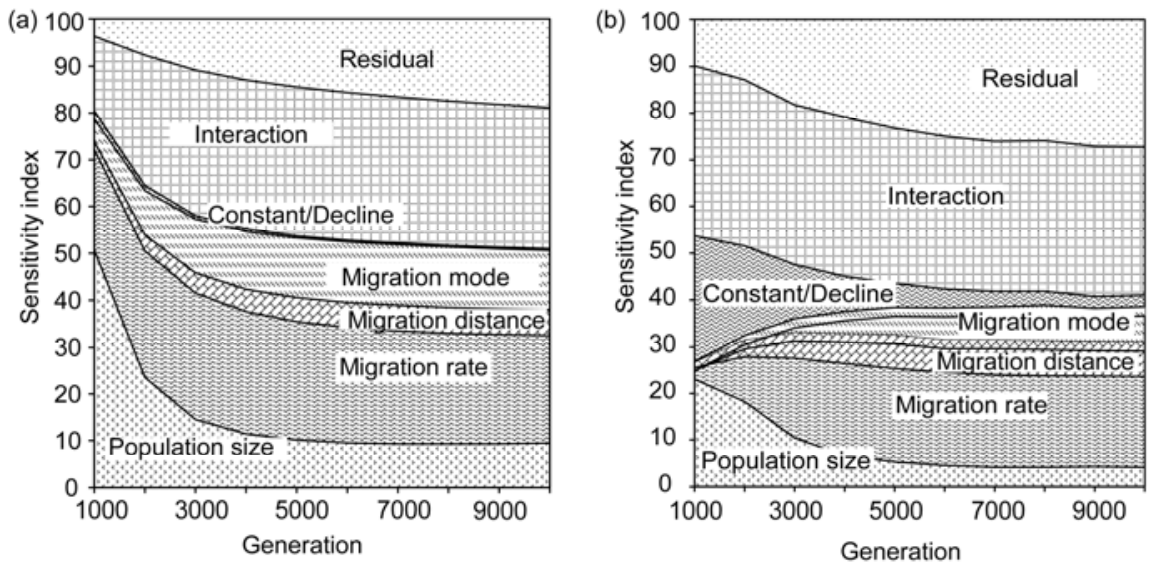

Figure 3 Time-dependent pie charts of (a) sensitivity indices for mean $H_{\mathrm{e}}$ and for (b) the mean slopes of linear regression on distance and $H_{\mathrm{e}}$. Interaction indicates the interactions between 2 parameters; residual indicates the interactions between 3 or more parameters. 
(a)

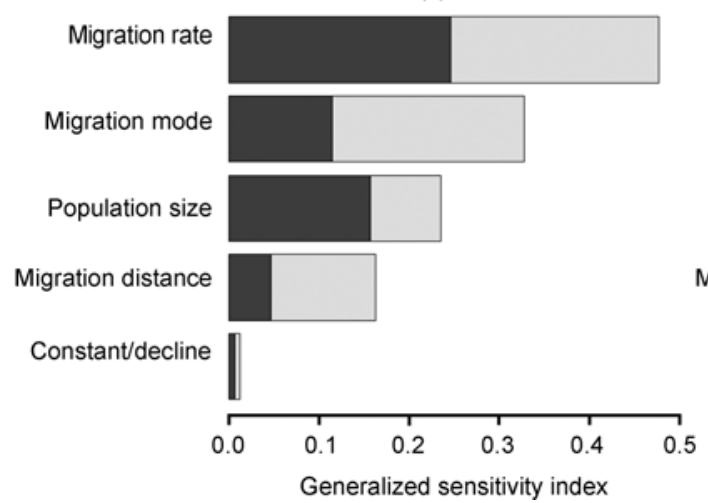

(b)

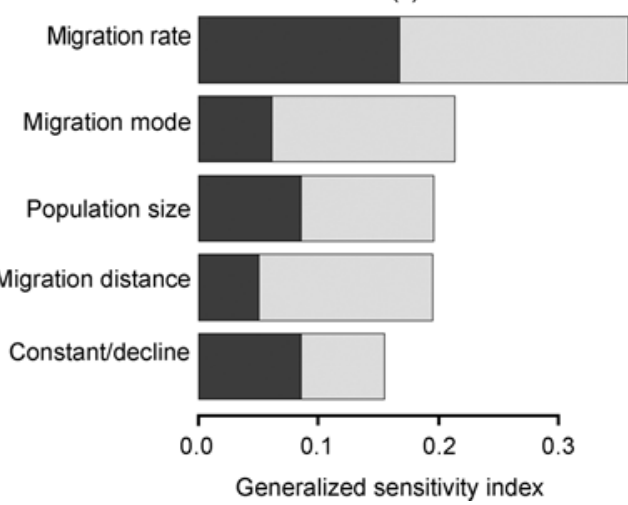

Figure 4 Generalized sensitivity indices of the 5 parameters for (a) mean $H_{\mathrm{e}}$ and (b) mean slopes of linear regression on distance and $H_{\mathrm{e}}$. The total length of a bar represents the total sensitivity index of a parameter. Black bars represent the main sensitivity indices; gray bars represent interaction sensitivity indices.

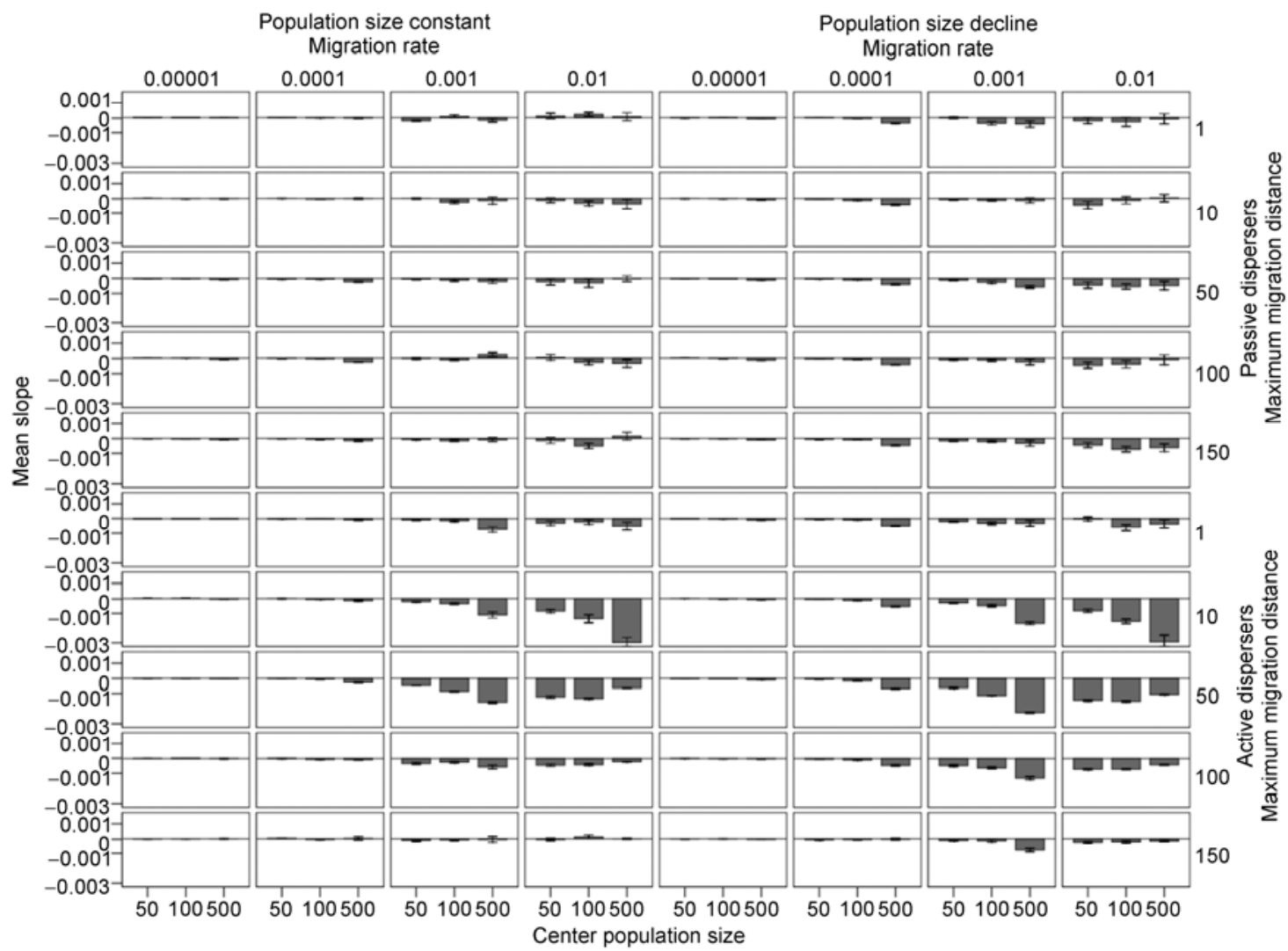

Figure 5 Mean slopes of linear regression on distance and $H_{\mathrm{e}}$ of 100 populations for each parameter combination after 10000 generations. Each simulation was repeated 10 times and data were regressed separately. Error bar shows \pm 1.0 standard error (SE).

(Figure 5). Active dispersers not only displayed a negative slope more frequently, but also had larger absolute values (strong correlation) than passive dispersers. Active dispersers with $m>0.001$ and moderate migration distances $\left(D_{\mathrm{m}}=\right.$ 10-100) demonstrated the most negative values, and the highest negative values of the slope occurred when $m$ $=0.001-0.01$ and $D_{\mathrm{m}}=10-50$ (Figure 5 ). When $D_{\mathrm{m}}=1$ or $D_{\mathrm{m}}>100$, the values decreased (Figure 5). This is consistent with the classic view of central-peripheral population dynamics [1,2]. A central population has more neighboring populations than a peripheral population does and, therefore, with an appreciable migration rate, a central population exchanges genes with more populations and maintains a higher $H_{\mathrm{e}}$ than a peripheral population does. However, when migration and gene flow are low, the difference of gene flow between a central population and a peripheral population is small and negligible. When maximum migration distance is low, for example $D_{\mathrm{m}}=1$, gene flow may only occur 
between populations in very close proximity and the benefit of being at a central location is limited. With the increase of $D_{\mathrm{m}}$, the benefit increases. However, when $D_{\mathrm{m}}$ is very high $\left(D_{\mathrm{m}}>100\right)$, many populations can reach each other and a peripheral population probably benefits from gene flow just as much as a central population. The difference between them, again, becomes negligible. The impact of migration on passive dispersers was different, particularly when the maximum migration distance was relatively high, e.g. $D_{\mathrm{m}}=$ 10-100 (Figure 5). A large number of migrants probably went outside the simulation area (and died), and therefore, did not contribute to gene flow. The gene flow experienced by the central and peripheral populations was probably very similar, which in turn, produced similar $H_{\mathrm{e}}$ values for both central and peripheral populations.

Population size and migration rate also demonstrated an interesting dynamic interaction. In the majority of cases, larger populations with higher migration rates tended to show negative slopes and larger negative values (Figure 5). This pattern was particularly noticeable for active dispersers. When population sizes were small and migration rates were low, both central and peripheral populations had a zero or near zero $H_{\mathrm{e}}$ (Figure 2). With the increased sizes, $H_{\mathrm{e}}$ of all populations would increase, but central populations would increase more than peripheral populations when there was an appreciable gene flow (migration). However, an opposite trend was revealed when migration was relatively strong or population size was relatively large. When $m=0.01$ and $D_{\mathrm{m}}$ $=50-100$, smaller populations produced a more negative slope (Figure 5). When $N_{\mathrm{e}}=500$ and $D_{\mathrm{m}}=50-100$, populations with a low migration rate $(m=0.001)$ produced a more negative slope than populations with a higher migration rate ( $m=0.01)$. This may be because the $H_{\mathrm{e}}$ values of both peripheral and central populations are sufficiently high and the difference between them is small when population sizes are relatively large and migration is sufficient (Figure 2). A population size of 500 and a migration rate of 0.01 have been considered as small and low [20,27,28]; however, our results demonstrated that these values are large enough to reduce the genetic difference between the central and peripheral populations (Figures 2 and 5).

A large number of combinations showed a slope of zero and there were two likely causes (Figure 5). First, for the parameter combinations with extremely low migration rates ( $m=0.00001-0.0001)$, all the populations finished with an extremely low $H_{\mathrm{e}}$ (close to zero; Figure 2). Consequently, the variation of $H_{\mathrm{e}}$ was too small to produce a significant value of the slope. Second, some population clusters may maintain relatively high $H_{\mathrm{e}}$ irrespective of their locations (Figure S1(a)). Populations at close proximity may maintain a relatively high gene flow among themselves even when the overall $m$ and $D_{\mathrm{m}}$ are small, and therefore, a cluster of neighboring populations may maintain high $H_{\mathrm{e}}$ despite an overall low $H_{\mathrm{e}}$ (Figure S1(a)). The location and formation of such high $H_{\mathrm{e}}$ population clusters are highly random and overall reduce the $H_{\mathrm{e}}$ difference between the central and peripheral populations. Populations are rarely distributed evenly in natural landscapes and population clusters are common. This observation may have important conservation implications, and population clusters with high $H_{\mathrm{e}}$ may deserve more conservation attention.

Sensitivity analysis provided further support to our assessment (Figures 2(b) and 3(b)). Although every parameter contributed significantly to the final outcome of the regression slope, individually, their interactions were responsible for more than $50 \%$ of the total variability. Again, drift (population size) related parameters were the most important for the first 4000 generations, and migration related parameters became more important later in the simulation.

\subsection{The impact of population establishment time}

Simulations with a central population size of 500 were more likely to produce a strong negative slope after 10000 generations (Figure 5). Therefore, we examined the impact of population establishment time on the slope value with a central population size of 500. The change of mean slope of linear regression of $H_{\mathrm{e}}$ and distance over generations is shown in Figure 6.

When peripheral populations were smaller than central populations, the mean slope quickly reached a peak negative value at approximately 1000 generations and then gradually reversed towards zero (Figure 6). This is likely because of a rapid decrease of $H_{\mathrm{e}}$ of the peripheral populations within the first 1000 generations caused by strong genetic drift. Because of large population sizes, the decrease of $H_{\mathrm{e}}$ of the central populations was slow and lagged behind that of the peripheral populations. This produced the large negative slope. The $H_{\mathrm{e}}$ of peripheral populations reached its minimum, in many cases zero, at around 1000 generations, but the $H_{\mathrm{e}}$ of central populations continued to decrease, resulting in a diminishing difference of $H_{\mathrm{e}}$ between the central and peripheral populations and the slope returning to zero (Figure 6). Nevertheless, when migration was relatively high (i.e. $m \geqslant 0.01, D_{\mathrm{m}} \geqslant 10$ ), the mean slope of active dispersers did not return to zero (Figure 6). The central populations of active dispersers were able to maintain high $H_{\mathrm{e}}$ with sufficient gene flow. Passive dispersers were unable to maintain high $H_{\mathrm{e}}$ even with the presence of relatively high migration, because a large number of migrants went out of the simulation area (and died), and did not contribute to gene flow. The largest negative slope was for an active disperser model with a relatively high migration rate $(m=0.01)$ and a moderate maximum migration distance $\left(D_{\mathrm{m}}=10\right)$ (Figures 5 and 6).

When the peripheral populations had the same sizes as the central populations, there was no major change during the first 1000 generations, and the mean slope either remained close to zero or gradually became negative (Figure $6)$. The active dispersers changed faster than passive 


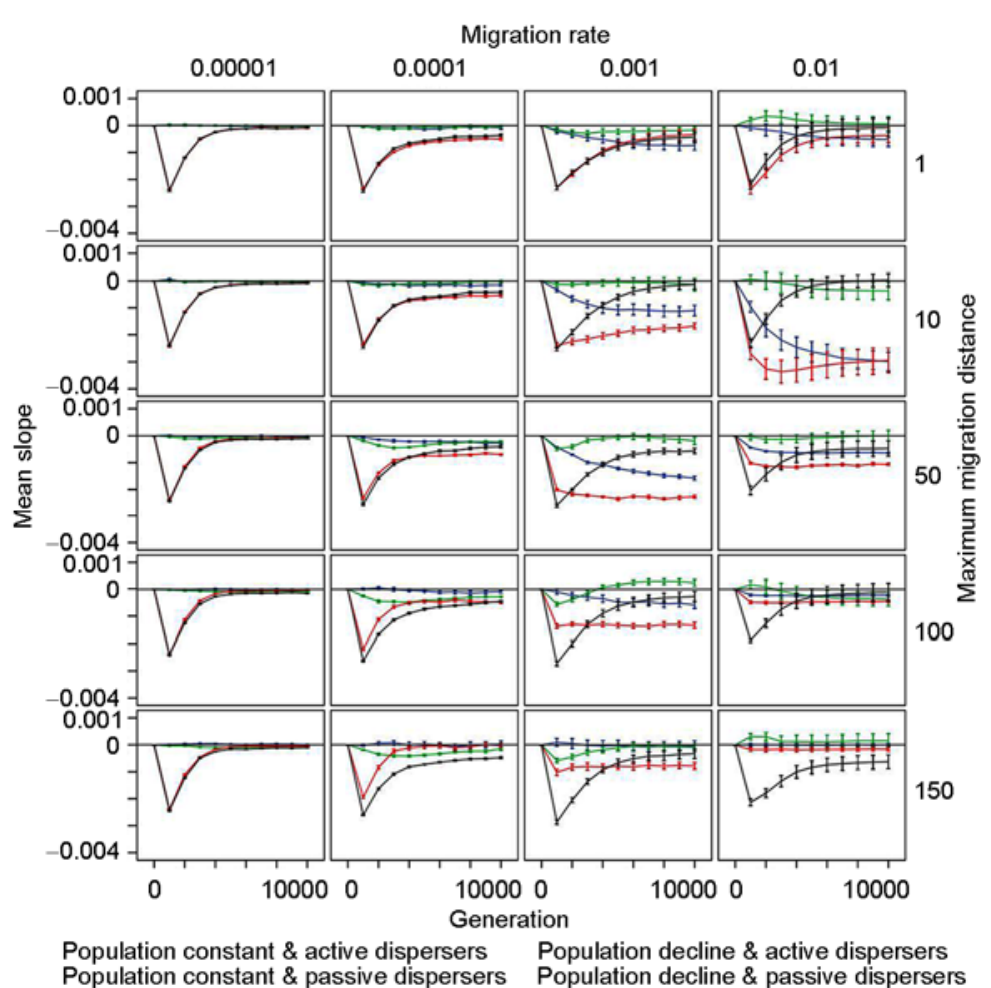

Figure 6 Change of mean slopes of linear regression on distance and $H_{\mathrm{e}}$ over generations with center population size $=500$. Each simulation was repeated 10 times.

dispersers, particularly when migration was moderately low ( $m=0.001-0.01$ and $\left.D_{\mathrm{m}}=10-50\right)$. When the migration was very low, gene flow could not maintain the $H_{\mathrm{e}}$ for either central or peripheral populations. Both reduced at a similar rate and the slope remained close to zero. When migration was moderately low, the gene flow was sufficient to maintain a high diversity for the central populations but was not enough for the peripheral populations. When migration was high, it homogenized all populations.

The mean slopes reached equilibrium after approximately 4000-6000 generations, while dramatic changes occurred only within the first 4000 generations. This is consistent with the results of the sequential global sensitivity analysis (Figure 3) in which the contribution of each parameter stabilized after 4000 generations. This result may have important implications for the temperate zone fauna and flora of North America and Europe, because they were impacted by the Pleistocene glaciation, of which, the latest was approximately 10000 years ago [29]. Species with short generation times have probably already reached equilibrium; species with long generation times may still be experiencing dramatic changes which, according to our simulation results, will make them more like to display the $\mathrm{CPH}$ pattern.

\section{Conclusion}

When should we expect higher genetic diversity in central populations? The majority of species probably do not show the pattern predicted by the central-peripheral population hypothesis. Species that possess certain properties (parameter combinations) are more likely to display the pattern than others. Active dispersers with a relatively low migration rate $(m)$ and a moderate maximum migration distance $\left(D_{\mathrm{m}}\right)$ are most likely to have higher genetic diversity in their central populations than in their peripheral populations. However, the interactions between the various parameters are likely to be more important than any single parameter. Furthermore, newly established populations are more likely to display the $\mathrm{CPH}$ pattern than well-established ones.

These conclusions have significant implications in conservation. To optimize cost benefits, should we attempt to conserve the largest populations within a species' range, or should we pay more attention to peripheral populations which are often perceived as fast evolving and having greater evolutionary potential $[2,19]$ ? The results from this study suggest that the difference in genetic diversity between the central and peripheral populations largely depends on the specific properties of the species in question. In some species, populations may have widely different genetic diversity and composition, while in others populations may have no differences at all. In addition, population clusters may possess high genetic diversity irrespective of their location, and therefore, may deserve more conservation attention. Furthermore, populations at different stages of establishment may be on very different trajectories and 
their genetic diversity may go up or down regardless of our conservation efforts. Using the refined parameters for the central-peripheral population hypothesis, a better evaluation for most species can probably be made, and an informed decision about where to set our conservation priorities and efforts may be possible.

This work was supported by the Knowledge Innovation Program of the Chengdu Institute of Biology, Chinese Academy of Sciences (CIB-2007LYQY-Q02) to $Q D$ and a Natural Science and Engineering Research Council of Canada (NSERC) Discovery Grant to JF.

1 Mayr E. Animal Species and Evolution. Cambridge: Harvard University Press, 1963

2 Lesica $\mathrm{P}$, Allendorf $\mathrm{F} \mathrm{W}$. When are peripheral populations valuable for conservation? Conserv Biol, 1995, 9: 753-760

3 Lesica P, Allendorf F W. Are small populations of plants worth preserving? Conserv Biol, 1992, 6: 135-139

4 Shumaker K M, Babbel G R. Patterns of allozyme similarity in ecologically central and marginal populations of Hordeumjubatum in Utah. Evolution, 1980, 34: 110-116

5 Gapare W J, Aitken S N, Ritland C E. Genetic diversity of core and peripheral Sitka spruce (Picea sitchensis (Bong.) Carr) populations: Implications for conservation of widespread species. Biol Conserv, 2005, 123: 113-123

6 Crozier R H. Genetic diversity and the agony of choice. Biol Conserv, 1992, 61: 11-15

7 Levin D A. Local speciation in plants: The rule not the exception. Syst Bot, 1993, 18: 197-208

8 Durka W. Genetic diversity in peripheral and subcentral populations of Corrigiola litoralis L. (Illecebraceae). Heredity, 1999, 83: 476484

9 Shea K L, Furnier G R. Genetic variation and population structure in central and isolated populations of balsam fir, Abies balsamea (Pinaceae). Am J Bot, 2002, 89: 783-791

10 Cassel A, Tammaru T. Allozyme variability in central, peripheral and isolated populations of the scarce heath (Coenonympha hero: Lepidoptera, Nymphalidae): Implications for conservation. Conserv Genet, 2003, 4: 83-93

11 Lönn M, Prentice H C. Gene diversity and demographic turnover in central and peripheral populations of the perennial herb Gypsophila fastigiata. Oikos, 2002, 99: 489-498

12 Zhan A, Li C, Fu J. Big mountains but small barriers: Population genetic structure of the Chinese wood frog (Rana chensinensis) in the Tsinling and Daba Mountain region of northern China. BMC Genetics, 2009, 10: 17

13 Guries R P, Ledig F T. Genetic diversity and population structure in pitch pine (Pinus rigida Mill). Evolution, 1982, 36: 387-402

14 Betancourt J L, Schuster W S, Mitton J B, et al. Fossil and genetic history of a pinyon pine (Pings edults) isolate. Ecology, 1991, 72: $1685-1697$

15 Garner T W J, Pearman P B, Angelone S. Genetic diversity across a vertebrate species' range: A test of the central-peripheral hypothesis. Mol Ecol, 2004, 13: 1047-1053

16 Garcia-Ramos G, Kirkpatrick M. Genetic models of adaptation and gene flow in peripheral populations. Evolution, 1997, 51:21-28

17 Griffin S R, Barrett S C H. Post-glacial history of Trillium grandiflorum (Melanthiaceae) in Eastern North America:Inferences from phylogeography. Am J Bot, 2004, 91: 465-473

18 Amato M, Brooks R J, Fu J. A phylogeographic analysis of populations of the wood turtle (Glyptemys insculpta) throughout its range. Mol Ecol, 2008, 17: 570-581

19 Lammi A, Siikamäki P, Mustajärvi K. Genetic diversity, population size, and fitness in central and peripheral populations of a rare plant Lychnis viscaria. Conserv Biol, 1999, 13: 1069-1078

20 Miller S D, MacInnes H E, Fewster R M. Detecting invisible migrants: An application of genetic methods to estimate migration rates. In: Thomson D L, Cooch E G, Conroy M J, eds. Modeling Demographic Processes in Marked Populations, Volume 3. New York: Springer, 2009. 417-437

21 Saltelli A, Chan K, Scott E M. Sensitivity Analysis. New York: Wiley, 2000

22 Dancik G M, Jones D E, Dorman K S. Parameter estimation and sensitivity analysis in an agent-based model of Leishmania major infection. J Theor Biol, 2010, 262: 398-412

23 Monod H, Naud C, Makowski D. Uncertainty and sensitivity analysis for crop models. In: Wallach D, Makowski D, Jones J W, eds. Working with Dynamic Crop Models: Evaluation, Analysis, Parameterization and Applications. Amsterdam: Elsevier, 2006. 55-100

24 Lamboni M, Makowski D, Monod H. Multivariate global sensitivity analysis for dynamic crop models. Field Crop Res, 2009, 113: 312-320

25 Lamboni M, Makowski D, Monod H. Multisensi: Multivariate Sensitivity Analysis. R package version 1.0-3. http: //CRAN.R-project.org/ package=multisensi, 2010.

26 R Development Core Team. R: A language and environment for statistical computing. Vienna: R Foundation for Statistical Computing, 2011

27 Lande R, Barrowclough G F. Effective population size, genetic variation, and their use in population management. In: Soulé M E, ed. Viable Populations for Conservation. Cambridge: Cambridge University Press, 1987. 87-123

28 Theodorou K, Couvet D. Genetic load in subdivided populations: Interactions between the migration rate, the size and the number of subpopulations. Heredity, 2006, 96: 69-78

29 Webb T, Bartlein P J. Global changes during the last 3 million years: Climatic controls and biotic responses. Ann Rev Ecol Syst, 1992, 23: $141-173$

Open Access This article is distributed under the terms of the Creative Commons Attribution License which permits any use, distribution, and reproduction in any medium, provided the original author(s) and source are credited.

\section{Supporting Information}

Figure S1 Population distribution in the hypothetical space and their $H_{\mathrm{e}}$ after 10000 generations. One of the 10 iterations. (a) Passive dispersers, population constant, size $=500, m=0.01, D_{\mathrm{m}}=150$; (b) active dispersers, population constant, size $=500, m=0.01, D_{\mathrm{m}}=150$; one of the 10 iterations.

The supporting information is available online at csb.scichina.com and www.springerlink.com. The supporting materials are published as submitted, without typesetting or editing. The responsibility for scientific accuracy and content remains entirely with the authors. 